\title{
Echo State Networks as Novel Approach for Low-Cost Myoelectric Control
}

\author{
* Cosima Prahm ${ }^{1}$, Alexander Schulz², Benjamin Paaßen², Oskar \\ Aszmann $^{1}$, Barbara Hammer ${ }^{2}$, and Georg Dorffner ${ }^{1}$ \\ ${ }^{1}$ Medical University of Vienna, Austria \\ ${ }^{2}$ Bielefeld University, Germany
}

(This is a preprint of the publication [15], as provided by the authors.)

\begin{abstract}
Myoelectric signals (EMG) provide an intuitive and rapid interface for controlling technical devices, in particular bionic arm prostheses. However, inferring the intended movement from a surface EMG recording is a non-trivial pattern recognition task, especially if the data stems from lowcost sensors. At the same time, overly complex models are prohibited by strict speed, data parsimony and robustness requirements. As a compromise between high accuracy and strict requirements we propose to apply Echo State Networks (ESNs), which extend standard linear regression with 1) a memory and 2) nonlinearity. Results show that both features, memory and nonlinearity, independently as well as in conjunction, improve the prediction accuracy on simultaneous movements in two degrees of freedom (hand opening/closing and pronation/supination) recorded from four able-bodied participants using a low-cost 8-electrode-array. However, it was also shown that the model is still not sufficiently resistant to external disturbances such as electrode shift.
\end{abstract}

\section{Introduction}

Robotic arm prostheses support upper limb amputees in everyday life tasks 3. Such prostheses are controlled through myoelectric signals derived from the patient's muscles in their residual limb. However, commercially established products are restricted to activating only a single degree of freedom (DoF) at a time and require tiresome mode-switching to execute movements in multiple DoFs [3]. More advanced prosthetic control systems acquire the user's muscle

*Funding by the DFG under grant number HA 2719/6-2, the CITEC center of excellence (EXC 277), and the Christian Doppler Research Foundation of the Austrian Federal Ministry of Science, Research and Economy is gratefully acknowledged. 
signals via an array of surface electrodes, infer the intended movement as well as the intended intensity via machine learning models and translate it to more natural prosthetic movements [7, 9, 17]. However, the requirements imposed on such a machine learning model are high: It should be complex enough to provide accurate prediction, but also require few patient training data, few electrodes, be robust to outside disturbances and provide real-time predictions with low delay [3. Especially electrode shift has been found to be a limiting factor in reliable prosthetic control, as it occurs every time the device is re-attached. Therefore, even though nonlinear regression techniques promise better recognition results, the strict requirements prohibit overly complex models [4]. So far, no machine learning model has yielded reliable enough predictions to be realized within a commercially available prosthesis.

In this contribution we analyzed whether extending a simple linear regression model [4] with two key features, namely memory (in time) and nonlinearity, offers significant improvement with respect to recognition accuracy without sacrificing too much in terms of speed and robustness. We applied Extreme Learning Machines (ELMs) [5] and Echo State Networks (ESNs) [6] to assess the benefit of nonlinearity and memory respectively. We evaluated the test recognition accuracy on undisturbed data as well as data which was disturbed by electrode shift. Our data set consisted of simultaneous movements in two DoFs from four able-bodied participants, recorded using the Thalmic Myo (Thalmic Labs, Canada) armband, a commercially availabe, low-cost 8-electrode array, which has already shown promising applications in the area of prosthetic training and control [8, 13].

\section{Methods}

\subsection{From Linear Regression to Echo State Networks}

We introduce Echo State Networks (ESNs) as a systematic extension of linear regression (LR) by nonlinearity and memory. Let $\vec{x}_{t} \in \mathbb{R}^{K}$ denote the input at time step $t$ (i.e. the $K$ features computed on from the myoelectric signal at time step $t$ ) and let $\vec{y}_{t} \in \mathbb{R}^{n}$ be the desired output at time $t$ (i.e. the desired movement in each of the $n$ degrees of freedom). We can write these as matrices of the form $X=\left(\vec{x}_{1}, \ldots, \vec{x}_{T}\right) \in \mathbb{R}^{K \times T}$ and $Y=\left(\vec{y}_{1}, \ldots, \vec{y}_{T}\right) \in \mathbb{R}^{n \times T}$. Then, linear regression assumes a direct, linear relationship between input- und output of the form $Y=W \cdot X$ for some matrix $W \in \mathbb{R}^{n \times K}$ which is set to $W=$ $Y \cdot X^{T} \cdot\left(X \cdot X^{T}\right)^{-1}$ in order to minimize the squared error [2].

We can equivalently describe linear regression as a feedforward neural network with a single hidden layer. Let $\vec{h}_{t} \in \mathbb{R}^{m}$ denote the value of the $m$ hidden layer neurons at time $t$. This value is computed as $\vec{h}_{t}=W^{\text {in }} \cdot \vec{x}_{t}$ for some input weight matrix $W^{\text {in }} \in \mathbb{R}^{m \times K}$. Similarly, the output of the network is computed

as $W^{\text {out }} \cdot \vec{h}_{t}$ for some output weight matrix $W^{\text {out }} \in \mathbb{R}^{n \times m}$. Due to linearity, the network output is fully described by the product matrix $W=W^{\text {out }} \cdot W^{\text {in }}$ which we can optimize as before. 
This analytical optimal solution is no longer possible if we introduce a nonlinearity in the hidden layer; that is, we compute the value of the hidden neurons as $\vec{h}_{t}=\sigma\left(W^{\text {in }} \cdot \vec{x}_{t}\right)$ where $\sigma()$ denotes the component-wise application of some nonlinear function $\sigma$, e.g. the tanh function. Here, one relies on gradient-based optimization methods such as backpropagation, which are prone to local optima. An alternative strategy is to not train the input weights $W^{\text {in }}$ at all, but to keep them fixed at some random value. Then, one can preprocess the input data via $H=\sigma\left(W^{\text {in }} \cdot X\right)$ and obtain an optimal solution for the output weights as in linear regression by setting $W^{\text {out }}=Y \cdot H^{T} \cdot\left(H \cdot H^{T}\right)^{-1}$. This is the Extreme Learning Machine (ELM) model, which has been shown to be a universal approximator with good generalization properties [5] and to be competitive with nonlinear classification models on EMG data [1].

Additionally, one can introduce a memory to the model, such that the value of the hidden layer neurons is computed dependent on the previous value of the hidden layer neurons giving rise to the equations

$$
\begin{aligned}
& \vec{h}_{t}=\sigma\left(W^{\text {in }} \cdot \vec{x}_{t}+W^{\text {hid }} \cdot \vec{h}_{t-1}\right) \\
& \vec{y}_{t}=W^{\text {out }} \cdot \vec{h}_{t}
\end{aligned}
$$

for some recurrent weight matrix $W^{\text {hid }} \in \mathbb{R}^{m \times m}$. Approximate analytical solutions for the weight matrices are provided by [12]. Alternatively, one can again set the recurrent weights $W^{\text {hid }}$ independently of the input (e.g. randomly) and train only the output weights via linear regression. Note that one also has to ensure that the recurrent weights $W^{\text {hid }}$ are set such that initial conditions vanish over time (echo state property [18]). Such models are called Echo State Networks (ESNs). If $\sigma$ is the identity, we call the model a linear Echo State Network (lESN). Here, we apply cycle reservoirs with jumps (CRJs) as introduced by [16]. In CRJs, input and reservoir weights are set deterministically to a single fixed value with varying signs according to an aperiodic, deterministic sequence. Hidden neurons are connected sparsely in a large cycle and additional jump connections between distant neurons within the cycle.

Given the strict runtime constraints of our application domain, fast models are desirable. Fortunately, ESNs have little complexity overhead compared to linear regression (provided that the number of hidden neurons $m$ is sufficiently small). For all models, a matrix inversion is required for training which has cubic complexity in the matrix dimension, i.e. $\mathcal{O}\left(K^{3}\right)$ for linear regression and $\mathcal{O}\left(\mathrm{m}^{3}\right)$ for all other models. Prediction requires one matrix multiplication in $\mathcal{O}(K \cdot n)$ for linear regression, two matrix multiplications in $\mathcal{O}(K \cdot m+m \cdot n)$ for ELMs and three matrix multiplications in $\mathcal{O}\left(K \cdot m+m^{2}+m \cdot n\right)$ for lESNs and ESNs, which reduces to $\mathcal{O}(K \cdot m+m \cdot n)$ in our case due to sparsity in $W^{\text {hid }}$.

\subsection{Experimental Protocol}

Four able-bodied participants executed a sequence of eight movements (hand open, hand close, pronation, supination, hand open + pronation, hand open + 
Table 1: The average classification error (between 0 and 1) across all movements for each degree of freedom (listed as rows) and each model (listed as columns). The standard deviation is provided in brackets. The top two rows show the results on the original data, the bottom two rows on the shifted data.

\begin{tabular}{|c|c|c|c|c|c|}
\hline condition & DoF $\backslash$ model & LR & ELM & IESN & ESN \\
\hline \multirow{4}{*}{ unshifted } & hand close/open & 0.158 & 0.072 & 0.105 & 0.042 \\
\hline & & $(0.171)$ & $(0.102)$ & $(0.203)$ & $(0.112)$ \\
\hline & pronation/supination & 0.186 & 0.069 & 0.116 & 0.050 \\
\hline & & $(0.176)$ & $(0.097)$ & $(0.183)$ & $(0.126)$ \\
\hline \multirow{4}{*}{ shifted } & hand close/open & 0.446 & 0.359 & 0.437 & 0.394 \\
\hline & & $(0.226)$ & $(0.168)$ & $(0.231)$ & $(0.248)$ \\
\hline & pronation/supination & 0.448 & 0.336 & 0.430 & 0.383 \\
\hline & & $(0.214)$ & $(0.129)$ & $(0.225)$ & $(0.245)$ \\
\hline
\end{tabular}

supination, hand close + pronation, and hand close + supination) ten times using their non-dominant hand. Each movement was executed between $3-5 s$. Afterwards, the electrode array was shifted for one electrode in medial direction around the forearm, and the movement sequence was recorded four times.

We recorded the myoelectric data with the Thalmic Myo armband using all 8 channels. For each channel, we computed the log-variance and the squared log-variance on windows of $120 \mathrm{~ms}$ with $40 \mathrm{~ms}$ overlap, resulting in $K=16$ features. We generated a separate output signal for both DoFs $(n=2)$ with three possible values each $(-1,0$, and 1 , i.e.: the output vector $(1,1)$ codes hand open + supination, the output vector $(1,0)$ codes hand open). We obtained a classification output from the regression output using simple thresholding $((-\infty,-0.5] \mapsto-1,(-0.5,0.5) \mapsto 0$, and $[0.5, \infty) \mapsto 1)$.

We evaluated the models in a leave-one-out crossvalidation over the 80 movements, optimizing hyperparameters via 1000 trials of random-search on the respective training data. The resulting model was then applied to the shifted data.

\section{$3 \quad$ Results}

The average classification error for the unshifted data is shown in the top two rows of Table 1. Using a Bonferrroni corrected Wilcoxon signed-rank test with $\alpha=0.001$ over all movements of all subjects $(N=320)$ we obtained the following significant differences: ELM is superior to linear regression (LR), IESN is superior to LR, ESN is superior to all three (LR, ELM and IESN).

After the electrode shift, a significant degradation of classification accuracy can be observed for all models, as given in the bottom two rows of Table 1 . 


\section{Discussion and Conclusion}

We have demonstrated that extending a linear regression model with nonlinear features and memory in the form of an Echo State Network (ESN) leads to significant improvements in terms of recognition accuracy on sensor data obtained from a low-cost electrode array for simultaneous movements in multiple degrees of freedom. Further, we have demonstrated that combining both features is superior to each single feature. As such, ESNs present a promising method for future research as well as clinical applications. However, we have also shown that ESNs are not sufficiently robust regarding external disturbances, namely electrode shift. Further work would be required to address this problem, e.g. using resistant features [11] or transfer learning [10, 14].

\section{References}

[1] Anam, K., Al-Jumaily, A.: Evaluation of extreme learning machine for classification of individual and combined finger movements using electromyography on amputees and non-amputees. Neural Networks 85, 51-68 (2017)

[2] Bishop, C.M.: Pattern Recognition and Machine Learning. Springer, Secaucus, NJ, USA (2006)

[3] Farina, D., Jiang, N., Rehbaum, H., Holobar, A., Graimann, B., Dietl, H., Aszmann, O.C.: The extraction of neural information from the surface emg for the control of upper-limb prostheses: Emerging avenues and challenges. IEEE Transactions on Neural Systems and Rehabilitation Engineering 22(4), 797-809 (2014)

[4] Hahne, J.M., Biebmann, F., Jiang, N., Rehbaum, H., Farina, D., Meinecke, F.C., Müller, K.R., Parra, L.C.: Linear and nonlinear regression techniques for simultaneous and proportional myoelectric control. IEEE Transactions on Neural Systems and Rehabilitation Engineering 22(2), 269-279 (2014)

[5] Huang, G.B., Zhu, Q.Y., Siew, C.K.: Extreme learning machine: Theory and applications. Neurocomputing 70(1-3), $489-501$ (2006)

[6] Jaeger, H., Haas, H.: Harnessing nonlinearity: Predicting chaotic systems and saving energy in wireless communication. Science 304(5667), 78-80 (2004)

[7] Jiang, N., Englehart, K.B., Parker, P.A.: Extracting simultaneous and proportional neural control information for multiple-dof prostheses from the surface electromyographic signal. IEEE Transactions on Biomedical Engineering 56(4), 1070-1080 (2009)

[8] Masson, S., Fortuna, F., Moura, F., Soriano, D., do ABC, S.B.d.C.: Integrating myo armband for the control of myoelectric upper limb prosthesis. 
Preprint of the publication [15], as provided by the authors.

Proceedings of the XXV Congresso Brasileiro de Engenharia Biomédica (2016)

[9] Ortiz-Catalan, M., Brånemark, R., Håkansson, B.: Biopatrec: A modular research platform for the control of artificial limbs based on pattern recognition algorithms. Source Code for Biology and Medicine 8(1), 1-18 (2013)

[10] Paaßen, B., Schulz, A., Hahne, J., Hammer, B.: An EM transfer learning algorithm with applications in bionic hand prostheses. In: ESANN (2017), accepted

[11] Pan, L., Zhang, D., Jiang, N., Sheng, X., Zhu, X.: Improving robustness against electrode shift of high density emg for myoelectric control through common spatial patterns. Journal of NeuroEngineering and Rehabilitation 12(1), 1-16 (2015)

[12] Pasa, L., Sperduti, A.: Pre-training of recurrent neural networks via linear autoencoders. In: NIPS. pp. 3572-3580 (2014)

[13] Phelan, I., Arden, M., Garcia, C., Roast, C.: Exploring virtual reality and prosthetic training. In: Virtual Reality (VR), 2015 IEEE. pp. 353-354. IEEE (2015)

[14] Prahm, C., Paassen, B., Schulz, A., Hammer, B., Aszmann, O.: Transfer learning for rapid re-calibration of a myoelectric prosthesis after electrode shift. In: ICNR2016, October 18-21, 2016, Segovia, Spain. pp. 153-157 (2016)

[15] Prahm, C., Schulz, A., Paaßen, B., Aszmann, O., Hammer, B., Dorffner, G.: Echo State Networks as Novel Approach for Low-Cost Myoelectric Control. In: ten Telje, A., Holmes, J.H., Sacchi, L., Popow, C. (eds.) Proceedings of the 16th Conference on Artificial Intelligence in Medicine (AIME 2017). vol. 10259. Springer, Vienna, Austria (2017)

[16] Rodan, A., Tiňo, P.: Simple deterministically constructed cycle reservoirs with regular jumps. Neural Computation 24(7), 1822-1852 (2012)

[17] Vujaklija, I., Farina, D., Aszmann, O.: New developments in prosthetic arm systems. Orthop. Res. Rev 8, 31-39 (2016)

[18] Yildiz, I.B., Jaeger, H., Kiebel, S.J.: Re-visiting the echo state property. Neural Networks 35, 1-9 (2012) 